\title{
La recombinaison homologue De nouvelles perspectives pour la transgénèse chez les mammifères
}

La recombinaison homologue permet l'échange de fragments d'ADN entre des double brins possédant des séquences identiques ; elle est en jeu dans l'intégration à son site chromosomique normal d'un gène ou d'une portion de gène introduit dans une cellule animale. Chez les mammifères, les événements de recombinaison homologue représentent environ 1/500 à 1/2000 des événements de recombinaison au hasard, si bien que des méthodes particulières doivent être mises en œuvre pour distinguer ces deux types d'intégration. Il est possible - quoique difficile - de créer des lignées d'animaux transgéniques par recombinaison homologue, le plus souvent par l'intermédiaire de cellules souches multipotentes dérivées de la masse interne de l'embryon. Cette technique est particulièrement prometteuse pour muter des gènes murins dont on désire évaluer les effets. A plus long terme, et à condition que la recombinaison soit vraiment « fidèle », une semblable approche pourrait déboucher sur une microchirurgie du gène dont des mutations délétères pourraient être corrigées exactement.

\section{Valérie Lemarchandel Xavier Montagutelli}

\section{ADRESSES}

V. Lemarchandel : boursière $M R T$. Inserm U 91, hôpital Henri-Mondor, 51, avenue du Maréchal-de Lattre de Tassigny, 94010 Créteil, France.

$\mathrm{X}$. Montagutelli : assistant. Unité de génétique des mammifères, Institut Pasteur, 25, rue

'introduction d'un gène étranger dans le patrimoine héréditaire d'un individu définit la transgénèse. Différentes techniques sont utilisées chez les mammifères pour y parvenir. Les deux premières ne permettent pas, à elles seules, de choisir l'endroit où le transgène s'intégrera. Il s'agit : (1) de la micro-injection dans l'œuf, qui permet d'introduire de grands fragments d'ADN exogène ; (2) des rétrovirus recombinants défectifs, qui permettent d'intégrer avec une fréquence élevée des fragments d'ADN dont la taille est cependant limitée par la place disponible dans la capside du rétrovirus.

En revanche, l'utilisation de cellules souches embryonnaires modifiées génétiquement in vitro puis réintro- duites dans un embryon receveur permet de réaliser un ciblage précis de la mutation souhaitée.

La manipulation génétique la plus fine que l'on puisse réaliser est la recombinaison homologue, c'est-àdire un événement au cours duquel les séquences d'un fragment d'ADN exogène viennent remplacer les séquences endogènes avec lesquelles elles présentent une homologie. Grâce à des astuces de biologie moléculaire, il est possible de sélectionner les cellules ES dans lesquelles le transgène est venu s'intégrer dans un endroit précis (à la base près).

\section{Les cellules ES}

Les cellules ES (embryonic stem cells, cellules souches embryonnaires) sont des cellules embryonnaires précoces 
totipotentes (c'est-à-dire capables de donner naissance à tous les types de tissus différenciés). Elles sont obtenues en mettant en culture des cellules de la masse cellulaire interne provenant d'embryons au stade préimplantatoire (blastocyste). Le maintien de la totipotentialité de ces cellules nécessite des conditions de culture particulières, notamment la présence d'un facteur de croissance, le LIF, récemment cloné [1], qui peut être apporté soit par adjonction de facteur purifié, soit par coculture avec des fibroblastes embryonnaires murins.

Il est possible d'injecter ces cellules dans la cavité d'un blastocyste receveur [2]. Environ $80 \%$ des embryons survivent à cette manipulation et jusqu'à $90 \%$ des survivants sont chimériques. La contribution des ES injectées dans la composition des différents tissus de ces chimères peut atteindre $90 \%$ [3].

Les cellules ES présentent au moins deux caractéristiques intéressantes qui les rendent aptes à participer à la constitution d'individus chimériques. Elles possèdent un fort potentiel de différenciation et une constitution chromosomique normale (au moins durant les premiers passages). Avant de procéder à l'injection de cellules ES dans un blastocyste, il est important de vérifier ces deux caractéristiques principales, surtout si les cellules ont fait l'objet de manipulations génétiques ou de repiquages répétés.

Pour obtenir des animaux transgéniques, il est préférable d'utiliser des mâles chimériques, que l'on fabrique en injectant des ES de constitution chromosomique $\mathrm{XY}$ dans des blastocystes. On obtient alors différents types d'animaux [3]

- Des mâles qui produisent des spermatozoïdes « de type ES » en faible pourcentage (de 0,3 à $3 \%$ maximum). Ils résultent de l'introduction de cellules ES (XY) dans un blastocyste (XY), les cellules introduites ayant participé, en proportion variable mais toujours faible, à la formation de la lignée germinale.

- Des mâles dont les spermatozoïdes dérivent exclusivement des cellules ES injectées. Ces mâles proviennent d'un blastocyste (XX) dans lequel $\mathrm{m} / \mathrm{s} n^{\circ} 1$ vol. 6 , janvier 90

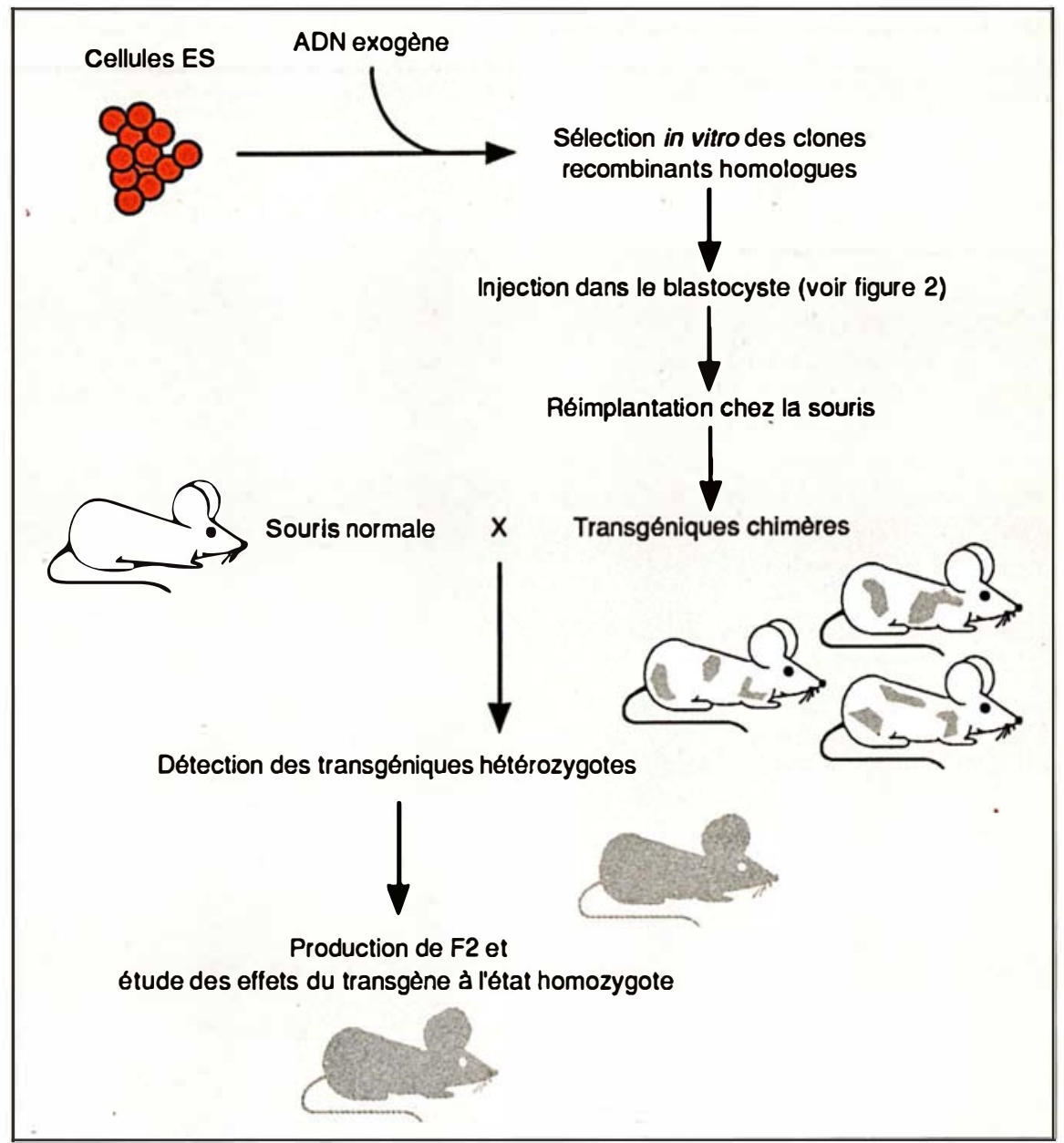

Figure 1. Transgénèse utilisant les cellules ES. Les cellules ES cultivées in vitro sont transfectées avec I'ADN exogène. Les clones recombinants homologues sont sélectionnés, puis injectés dans des blastocystes receveurs (voir figure 2). Les cellules ES proviennent d'une lignée présentant un pelage agouti (gris) et les blastocystes d'une lignée albinos (blanche) ou non-agouti (noire). Les mâles chimériques (qui présentent des taches grises sur un pelage blanc ou noir) sont accouplés avec des femelles de la même lignée que celle qui a fourni les blastocystes. Les individus hétérozygotes ainsi obtenus sont gris car le caractère agouti est dominant, alors que les caractères albinos et non-agouti sont récessifs. Les hétérozygotes sont croisés entre eux afin d'observer les effets du gène modifié à l'état homozygote.

les cellules ES introduites ont induit l'évolution de la gonade vers le sexe mâle (par l'intermédiaire de facteurs diffusibles). Seules les cellules (XY), donc «de type ES», peuvent participer à la spermatogenèse.

- Des femelles, ainsi que des mâles stériles dont certains présentent des anomalies du tractus génital. Ils résultent, eux aussi, de l'introduction de cellules ES (XY) dans un blasto- cyste $(\mathrm{XX})$, mais la proportion des cellules (XY) dans l'ébauche gonadique était trop faible pour induire son évolution complète vers le sexe mâle. Il est facile d'imaginer le moyen d'utiliser les cellules ES dans un protocole de transgénèse (figure 1 ). Les cellules doivent d'abord subir, in vitro, les manipulations génétiques souhaitées ainsi que les étapes de sélection correspondantes. Il faut ensuite vérifier qu'elles ont conservé 
leur totipotentialité et un caryotype normal. Alors seulement, elles peuvent être injectées dans des blastocystes. Les cellules ES et les blastocystes proviennent de deux lignées de souris qui diffèrent pour un gène de couleur de pelage (figures 1 et 2 ).

Parmi les individus chimériques, il faut sélectionner les mâles fertiles que l'on croise avec une femelle portant le même marqueur de pelage que les souris ayant fourni les blastocystes receveurs. Les descendants hétérozygotes « $\mathrm{ES} /+$ » sont facilement repérés par leur pelage et peuvent être accouplés entre eux pour obtenir des individus « ES/ES ».

\section{Les stratégies de sélection des recombinants homologues}

La plupart des travaux actuellement publiés sur la recombinaison homologue dans les cellules de mammifères ont utilisé des plasmides linéarisés introduits dans les cellules soit par électroporation (voir glossaire, p. 21), soit par micro-injection et plus rarement par coprécipitation de l'ADN avec du phosphate de calcium.

Caractérisation directe. La technique la plus directe, mais aussi la plus lourde, consiste à détecter les recombinants homologues par Southern blot sur l'ADN des cellules transfectées. C'est cette démarche que le groupe de Smithies a adoptée en 1985 [5] pour introduire, au locus de la $\beta$-globine, un gène $\beta$-globine comportant une délétion. Le plasmide utilisé comportait, outre le gène partiellement délété, le gène bactérien de résistance à la néomycine (néo), qui confère la résistance à une drogue (le G418) et peut servir à sélectionner les transfectants dans les cellules érythroïdes. L'ADN provenant de groupes de cellules résistantes au G418 a été hybridé avec une sonde spécifique du gène $\beta$ pour rechercher la présence du gène modifié introduit.

Cette technique de sélection est universelle et utilisable même lorsque la région non homologue est de taille réduite. Elle est toutefois très lourde à mettre en œuvre et elle a toujours été associée, par la suite, à des stratégies d'enrichissement cellulaire des $\checkmark$ Figure 2. Injection de cellules embryonnaires dans la cavité d'un blastocyste de souris [4]. A: une pipette de large diamètre permet de maintenir le blastocyste grâce à une légère aspiration. Une pipette plus fine contient les cellules embryonnaires à injecter. B : la pipette d'injection a été introduite dans la cavité du blastocyste. C: les cellules embryonnaires qui ont été injectées sont au contact des cellules de la masse cellulaire interne, avec lesquelles elles vont participer au développement de l'embryon. (Clichés Institut Pasteur.)

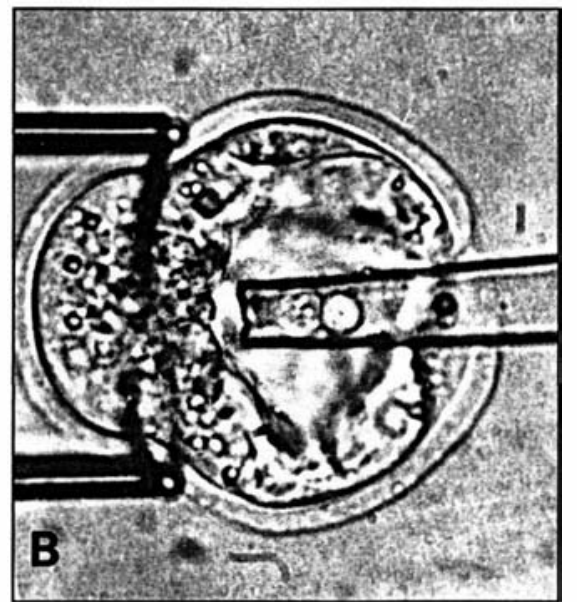

Sélection sur le gène cible. Il est parfois possible de sélectionner directement les recombinants lorsque la modification du gène cible fait apparaître un caractère de résistance à une drogue. Ce mode de sélection a été initialement appliqué au gène bactérien néo, qui confère la résistance au G418.

Après avoir établi plusieurs lignées cellulaires en transfectant des fibroblastes murins avec un gène néo portant soit une mutation ponctuelle, soit une délétion, Thomas et al. [6, 7] ont corrigé la mutation présente dans chaque lignée en micro-injectant un gène néo portant une mutation différente de celle présente dans la cellule receveuse et en sélectionnant par le G418 les cellules injectées. Dans les cellules résistantes au G418 (1/1000 environ), la structure du gène néo endogène est corrigée par recombinaison avec le gène néo plasmidique. De plus, le taux de recombinants obtenu ne dépend ni du nombre de copies injectées, ni de la localisation chromosomique du gène néo.

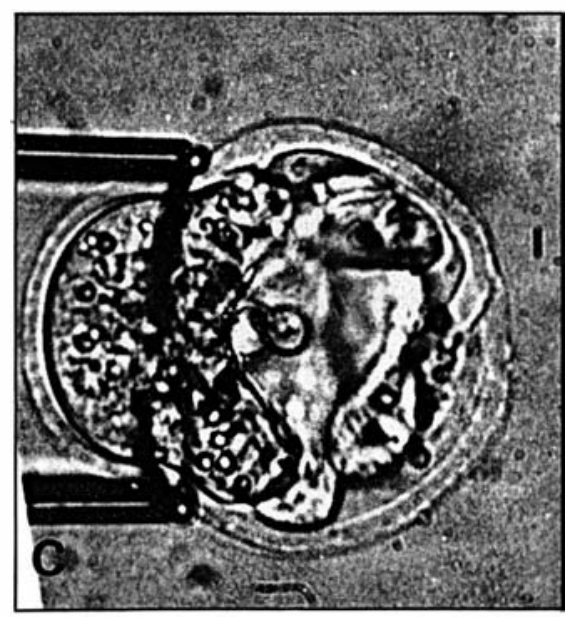

Song et al. [8] ont réalisé des expériences similaires sur des cellules humaines, les gènes néo (intégrés et transfectés) ayant dans ce cas des délétions différentes.

Une modification directe du gène Hprt (hypoxanthine phosphoribosyl transférase), pour lequel il est possible de sélectionner les cellules possédant cette fonction (avec le milieu HAT : hypoxanthine-aminoptérinethymidine) ou au contraire les cellules qui l'ont perdue (avec la 6-TG : 6-thioguanine) peut aussi être obtenue. De plus, le gène $\mathrm{Hprt}$ étant 
localisé sur le chromosome $\mathrm{X}$, il suffit d'en inactiver une seule copie, dans des cellules XY, pour obtenir un phénotype sélectionnable.

Doetschman et al. [9] ont par exemple corrigé une délétion de $10 \mathrm{~kb}$ touchant les deux premiers exons du gène $H$ prt présent dans une lignée de cellules ES. Pour ce faire, un plasmide, contenant les trois premiers exons de $\mathrm{Hprt}$, a été introduit par électroporation. Des clones recombinants sélectionnés en milieu HAT ont été obtenus à raison d'environ $1,4 \times 10^{-6}$ par cellule traitée.

Thomas et Capecchi [10] ont au contraire muté le gène $H$ prt dans des cellules ES en utilisant deux types de construction déduits des mécanismes de recombinaison connus chez la levure: des vecteurs de remplacement, dont les séquences viennent remplacer les séquences homologues endogènes, et des vecteurs d'insertion, dont les séquences s'insèrent au milieu des séquences endogènes (figure 3).
Les plasmides utilisés, introduits par électroporation, comportaient les exons 6 à 9 du gène $H$ prt, l'exon 8 étant interrompu par le gène néo. Dans un premier temps, les cellules ayant intégré le plasmide ont été sélectionnées par addition de G418 (environ une cellule résistante sur

\section{* GLOSSAIRE *}

Électroporation. Technique d'introduction de matériel étranger (par exemple d'ADN) dans une cellule, au cours de laquelle une décharge électrique est appliquée dans une petite cuve contenant le matériel étranger mélangé à la suspension cellulaire. Le choc électrique crée des pores dans la membrane cellulaire, permettant la pénétration par diffusion passive du matériel étranger.
1 000). Dans un second temps, celles qui ont subi une inactivation du gène $\mathrm{Hprt}$ ont été sélectionnées par addition de G-TG : entre une cellule pour 20000 et une cellule pour 950 , selon la longueur de la région d'homologie, ont été résistantes. Les deux types de vecteurs (de remplacement ou d'insertion) ont permis d'inactiver le gène $\mathrm{Hprt}$ avec la même efficacité.

Thomson et al. [11] ont réalisé un travail similaire à celui de Doetschman et al. [9] et obtenu, après injection des cellules ES porteuses du gène $\mathrm{Hprt}$ corrigé dans des blastocystes receveurs, des mâles chimériques puis, après croisement de ces mâles avec des femelles déficientes en HPRT, des souris exprimant le gène $H$ prt corrigé avec la même répartition tissulaire que des souris normales. C'est actuellement le seul travail qui démontre, grâce à l'obtention d'animaux hétérozygotes, que les cellules ES issues de ce type d'expérience peuvent coloniser

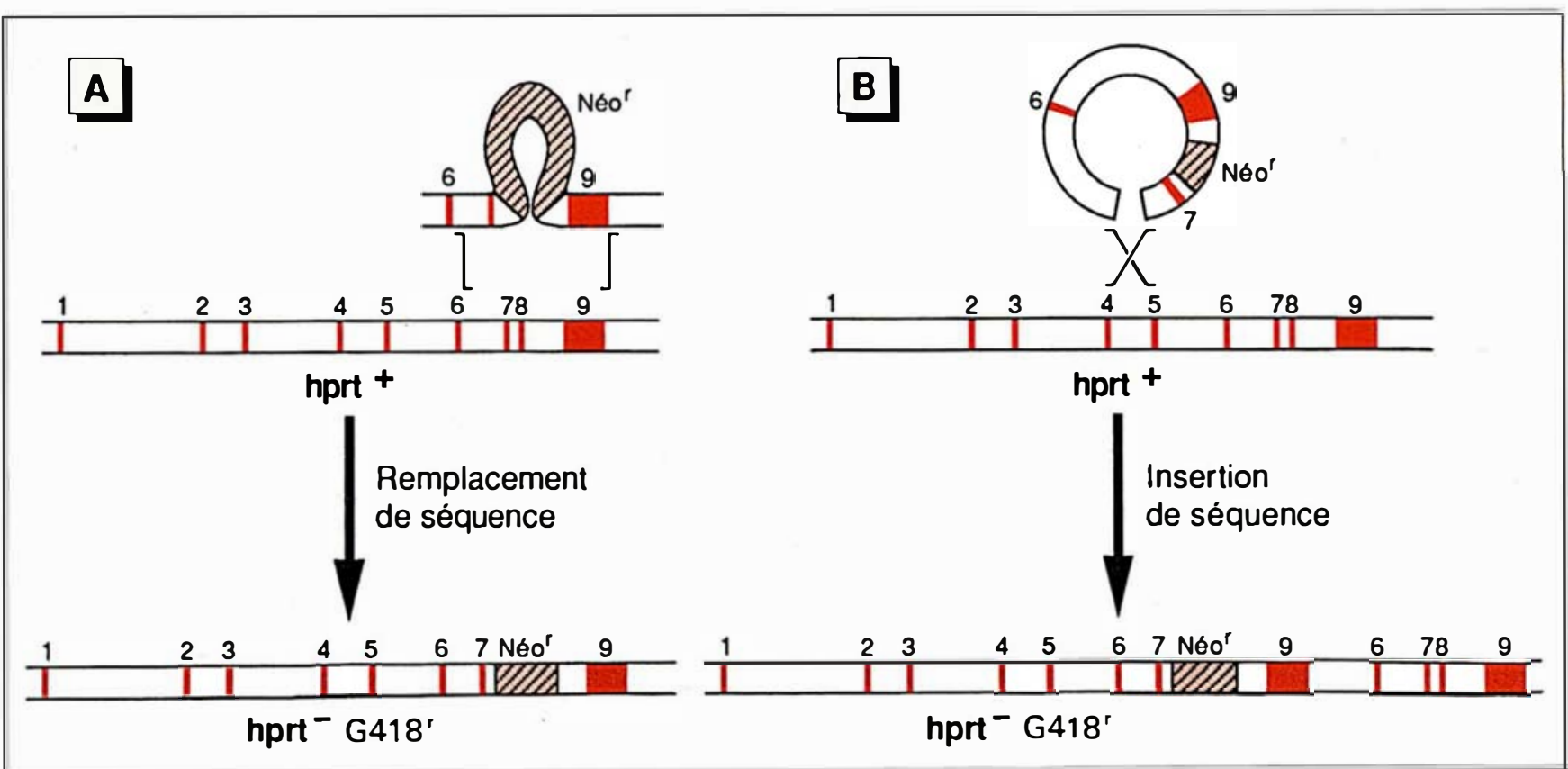

Figure 3. Vecteurs de remplacement et vecteurs d'insertion. A : vecteur de remplacement. Les séquences exogènes sont colinéaires des séquences endogènes et viennent les remplacer. B: vecteur d'insertion. Les séquences exogènes s'insèrent dans le gène cible et provoquent une duplication partielle des séquences homologues. Dans les deux cas, la séquence du gène cible est interrompue et la cellule acquiert le caractère sélectionnable - ici, la résistance au G418 conférée par le gène bactérien de résistance à la néomycine. (D'après [10].) 


\section{RÉFÉRENCES}

1. Williams RL, Hilton DJ, Pease S, et al. Myeloid leukemia inhibitory factor maintains the developmental potential of embryonic stem cells. Nature 1988 ; 336 : 684-7.

2. Gossler A, Doetschman T, Korn R, Serfling E, Kemler R. Transgenesis by means of blastocyst-derived embryonic stem cell lines. Proc Natl Acad Sci USA 1987 ; 83 : 9065-9.

3. Robertson EJ. Pluripotential stem cell lines as a route into the mouse germ line. Trends Genet 1986; $2:$ 9-13.

4. Babinet C. A simplified method for mouse blastocyst injection. Exp Cell Res $1980 ; 130$ : $15-9$.

5. Smithies O, Gregg RG, Boggs SS, Koralewski MA, Kucherlapati RS. Insertion of DNA sequences into the human chromosomal $\beta$-globin locus by homologous recombination. Nature $1985 ; 317: 230-4$.

6. Thomas KR, Folger KR, Capecchi MR. High frequency targeting of genes to specific sites in the mammalian genome. Cell 1986 ; 44 : 419-28.

7. Thomas KR, Capecchi MR. Introduction of homologous DNA sequences into mammalian cells induces mutations in the cognate gene. Nature 1986 ; 324 : $34-8$

8. Song KY, Schwartz F, Maeda N, Smithies $\mathrm{O}$, Kucherlapati R. Accurate modification of a chromosomal plasmid by homologous recombination in human cells. Proc Natl Acad Sci USA 1987 ; 84 : 6820-4.

9. Doetschman T, Gregg RG, Maeda N, et al. Targeted correction of a mutant $\mathrm{Hprt}$ gene in mouse embryonic stem cells. Nature 1987 330 : $576-8$.

10. Thomas KR, Capecchi MR. Site-directed mutagenesis by gene targeting in mouse embryo-derived stem cells. Cell $1987 ; 51$ : 50312.

11. Thompson S, Clarke AR, Pow AM, Hooper ML, Melton DW. Germ line transmission and expression of a corrected $\mathrm{Hprt}$ gene produced by gene targeting in embryonic stem cells. Cell 1989 ; 56 : 313-21.

12. Mansour SL, Thomas KR, Capecchi MR. Disruption of the proto-oncogene int-2 in mouse embryo-derived stem cells: a general strategy for targeting mutations to non-selec- la lignée germinale*.

Cette stratégie de sélection sur le gène cible, si elle est la plus simple à mettre en œuvre, est d'un domaine d'application extrêmement limité puisque peu de gènes conduisent, lorsqu'ils sont mutés, à un phénotype directement sélectionnable. Par ailleurs, l'inactivation d'un gène par cette méthode suppose qu'il soit représenté à un seul exemplaire dans le génome: ce n'est le cas que pour

* Note de la rédaction: depuis la rédaction de cet article, un petit nombre de transmissions à la descendance d'un transgène introduit par recombinaison homologue de cellules ES ont été rapportés dans des congrès, voire publiés (Science 1989; 246 : 799-803). (Nature 1989; 342: 435-8) (Proc Natl Acad Sci USA 1989 $86: 8927-31)$. les gènes portés par les chromosomes $\mathrm{X}$ et $\mathrm{Y}$.

Double sélection : positive et négative. Capecchi et al. [13] ont mis au point une méthode originale pour sélectionner directement les recombinants homologues, quel que soit le gène cible. La figure 4 présente la construction utilisée et les résultats escomptés.

Le plasmide contient des séquences du gène cible $X$, interrompues par le gène néo placé sous le contrôle d'un promoteur très fort (pour être exprimé quel que soit le site d'intégration). L'originalité de cette stratégie réside dans la présence du gène de la thymidine kinase du virus de l'Herpes simplex (Hsv-tk, sous le contrôle du même promoteur) à

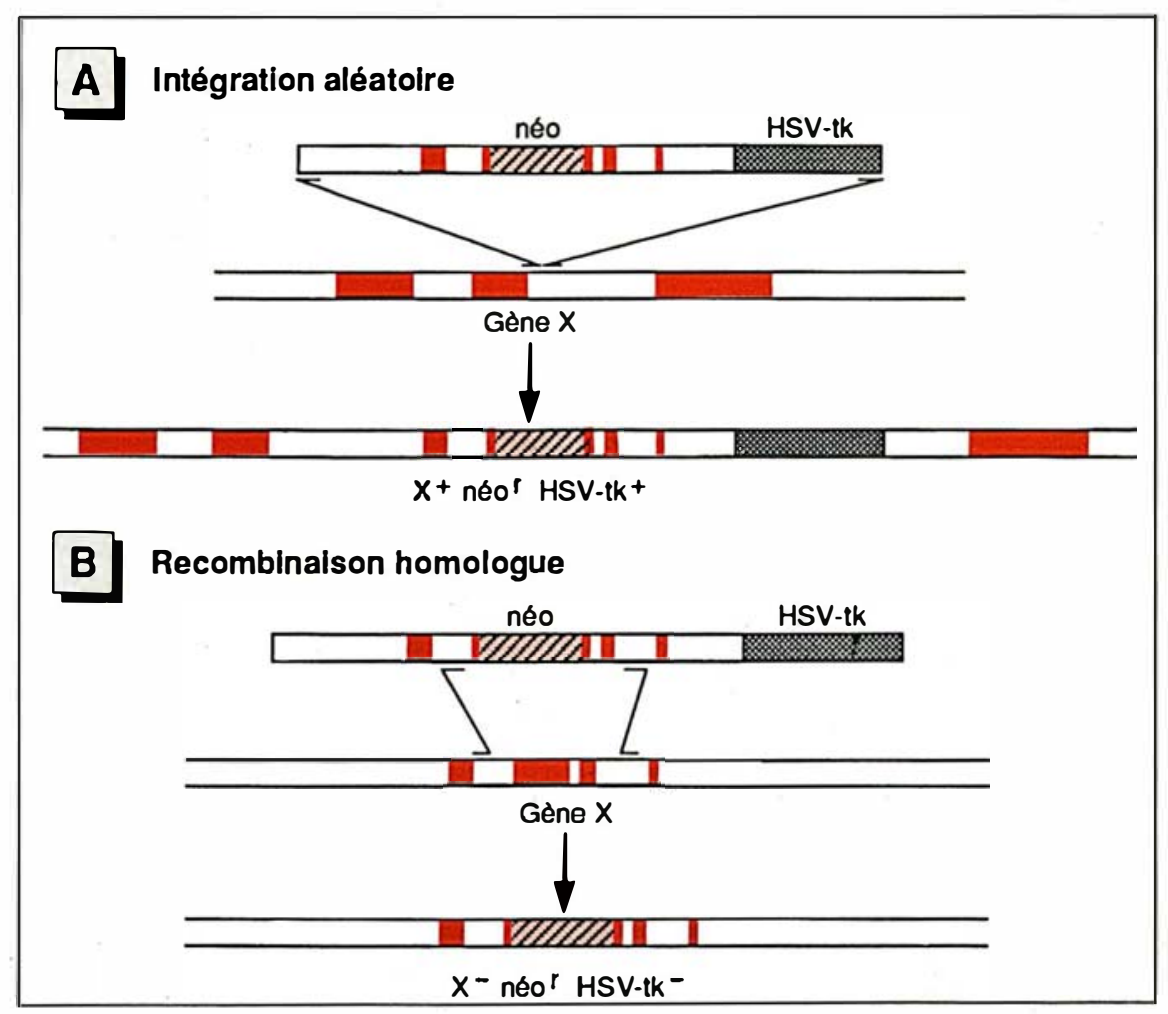

Figure 4. Sélection positive et négative. A : l'intégration aléatoire du plasmide a lieu par ses extrémités et concerne donc la totalité des séquences introduites. La cellule, qui possède les deux gènes néo et Hsv-tk, devient résistante au G418 mais elle est détruite par le ganciclovir. B : lors d'un événement de recombinaison homologue, le gène néo est intégré lors du remplacement des séquences endogènes par les séquences exogènes; le gène Hsv-tk n'est pas intégré car il ne contient pas de région d'homologie avec le gène cible. La cellule devient donc résistante au G418 et n'est pas détruite par le ganciglovir. Le gène X a été inactivé. (D'après [12].) 
l'une des extrémités du plasmide. Il confère aux cellules qui l'expriment la sensibilité à une drogue antivirale, le ganciclovir.

L'intégration aléatoire du plasmide a lieu par ses extrémités et concerne donc la totalité des séquences introduites. La cellule, qui possède les deux gènes néo et Hsu-tk, devient résistante au G418 mais elle est détruite par le ganciclovir.

Lors d'un événement de recombinaison homologue, le gène néo est intégré au cours du remplacement des séquences endogènes par les séquences exogènes ; en revanche, le gène $H s u$-tk n'est pas intégré car il ne contient pas de région d'homologie avec le gène cible. La cellule devient donc résistante au G418 et n'est pas détruite par le ganciclovir. Le gène $X$ a été inactivé.

Capecchi et al. [13] ont appliqué cette stratégie pour inactiver le gène $\mathrm{Hprt}$ d'une part, le proto-oncogène int-2 d'autre part. La sélection par le ganciclovir a permis d'enrichir d'un facteur 2000 la population de cellules résistantes au G418 en cellules ayant subi un événement de recombinaison au locus. Dans 19 cellules sur les 24 ainsi obtenues pour $\mathrm{Hprt}$, le gène a été inactivé. Le résultat est moins bon pour int-2 : 4 sur 81 . Les auteurs attribuent en partie cette différence à un moindre niveau d'expression puisque l'on retrouve moins d'un transcrit d'int-2 par cellule ES, à l'inverse de Hprt, exprimé à un niveau beaucoup plus élevé.

Cette stratégie réalise un enrichissement très efficace et elle est applicable facilement à n'importe quel gène cible. Elle est actuellement la seule technique capable d'exclure, grâce à la sélection négative, les cellules ayant subi, en plus d'une recombinaison homologue, l'intégration aléatoire d'une ou plusieurs copies de l'ADN exogène. Elle présente cependant l'inconvénient d'introduire un gène étranger dans le génome receveur. Elle a été appliquée pour inactiver des homéogènes impliqués au cours du développement : Hox 1.2 et Hox 1.3 [13].

Amplification génique (PCR). L'amplification enzymatique d'ADN in vitro (polymerase chain reaction: PCR) constitue une méthode sensible pour identifier les cellules qui possèdent la modification génétique $\mathrm{m} / \mathrm{s} n^{\circ} 1$ vol. 6 , janvier 90

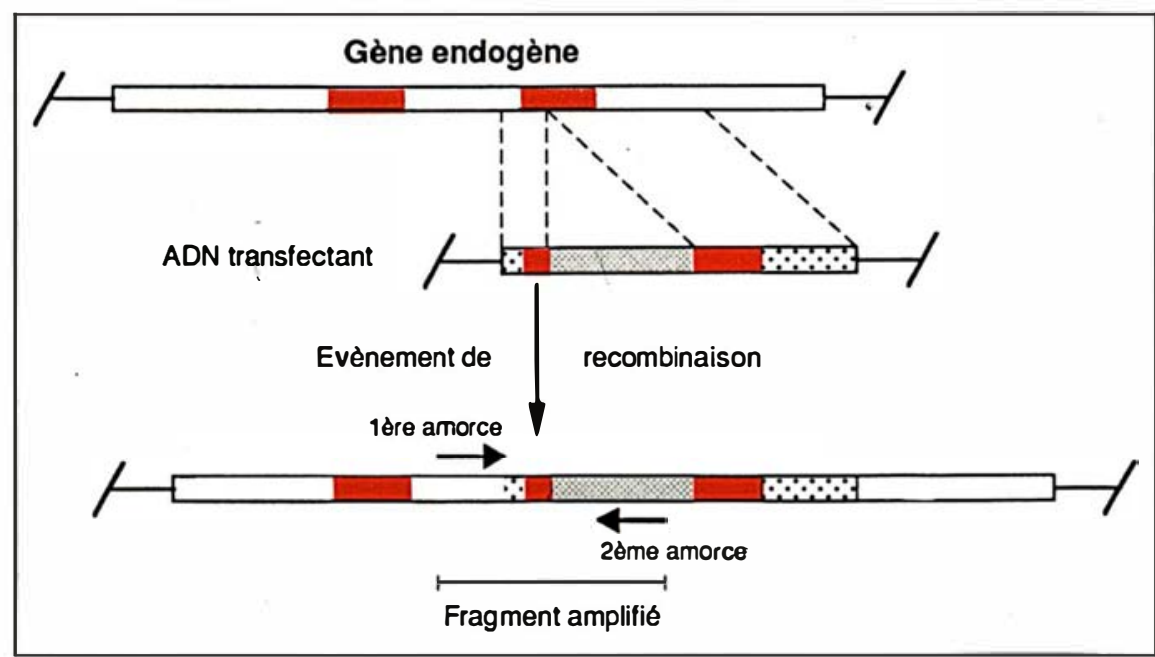

Figure 5. Détection des recombinants homologues par amplification génique. L'ADN extrait d'un groupe de cellules transfectées est mis en présence de deux amorces : l'une spécifique de séquences du gène situées à l'extérieur de la région d'homologie, l'autre spécifique de la modification introduite par I'ADN transfectant. La réaction d'amplification n'a lieu que lorsque I'ADN exogène s'est inséré dans la séquence endogène.

attendue, en particulier lorsque le gène altéré ne peut être soumis à aucune sélection directe ou indirecte. Cette détection ne dépend ni du phénotype induit par le gène modifié, ni de l'expression de ce gène dans la cellule cible. De plus, la taille de la séquence non homologue portée par l'ADN exogène peut être réduite à quelques nucléotides, ce qui pourrait favoriser la recombinaison homologue.

Le principe de détection du fragment recombinant est illustré sur la figure 5. L'ADN extrait d'un groupe de cellules transfectées est mis en présence de deux amorces : l'une spécifique de séquences du gène situées à l'extérieur de la région d'homologie, l'autre spécifique de la modification introduite par l'ADN transfectant. La réaction d'amplification n'a lieu que lorsque l'ADN exogène s'est inséré dans la séquence endogène. Cette technique permet de détecter une cellule modifiée parmi 1000 à 10000 cellules intactes. Elle est cependant d'une mise en ouvre délicate et de nombreuses précautions doivent être prises pour éviter les amplifications non spécifiques dues aux contaminations.

Deux équipes ont appliqué cette stratégie avec succès. Zimmer et al. [14] ont mutagénisé l'homéogène murin $\operatorname{Hox} 1.1$. La construction micro-injectée comporte un court oligonucléotide ( 20 paires de bases) qui interrompt la séquence codante de l'homéogène. Les auteurs ont détecté un recombinant homologue parmi 150 cellules traitées. Ils ont réinjecté des cellules ES ainsi modifiées dans des blastocystes receveurs et obtenu des souris chimériques.

Joyner et al. [15] ont su isoler trois lignées de cellules ES dans lesquelles une copie de l'homéogène $E n-2$ a été altérée par recombinaison homologue. Le locus endogène a été remplacé par une séquence comportant le gène procaryote néo sous le contrôle du promoteur du gène de la $\beta$-actine humaine. Par PCR (polymerase chain reaction), les auteurs ont détecté un événement d'intégration ciblée pour 300 colonies résistantes au G418.

Utilisation d'un gène de résistance sans promoteur. Une autre technique d'enrichissement cellulaire a été utilisée pour des gènes cibles transcrits dans les cellules ES.

Le vecteur utilisé comporte les séquences homologues du gène cible, au milieu desquelles est inséré le gène néo privé de son promoteur. En 


\begin{tabular}{|c|c|c|c|c|c|}
\hline \multicolumn{6}{|c|}{$\begin{array}{l}\text { Tableau I } \\
\text { VEC LES DIFFÉRENTES STRATÉGIES }\end{array}$} \\
\hline Système de sélection & Cellules & Gene cible & Expression & Caractère sélectionné & $\begin{array}{l}\text { Taux de recombinants homologues } \\
(\mathbf{R H})^{*}\end{array}$ \\
\hline $\begin{array}{l}\text { Caractérisation directe } \\
\text { Smithies et al. [5] }\end{array}$ & $\begin{array}{l}\text { EJ } \\
\text { Hu11 }\end{array}$ & $\begin{array}{l}\beta \text {-globine } \\
\beta \text {-globine }\end{array}$ & $\begin{array}{l}\text { Non } \\
\text { Oui }\end{array}$ & $\begin{array}{c}\text { aucun } \\
\text { néo+ : G418 + Southern }\end{array}$ & $\begin{array}{c}1 / 400 \text { à } 1 / 1500 \text { des } G 418^{r} \text { estimées } \\
1 / 300 \text { à } 1 / 1100 \text { des } G 418^{r}\end{array}$ \\
\hline $\begin{array}{l}\text { Sélection sur le gène cible } \\
\text { Thomas et al. [6] } \\
\text { Song et al. [8] } \\
\text { Doetschman et al. [9] } \\
\text { Thompson et al. [11] } \\
\text { Thomas et al. [10] }\end{array}$ & $\begin{array}{l}\text { LM } \\
\text { V79/EJ } \\
\text { ES } \\
\text { ES } \\
\text { ES }\end{array}$ & $\begin{array}{l}\text { néomut } \\
\text { néodel } \\
\text { hprt- } \\
\text { hprt- } \\
\text { hprt }\end{array}$ & $\begin{array}{l}\text { Oui } \\
\text { Oui } \\
\text { Oui } \\
\text { Oui } \\
\text { Oui }\end{array}$ & $\begin{array}{c}\text { néo }{ }^{+}: \text {G418 } \\
\text { néo }: \text { G418 } \\
\text { hprt }^{+}: \text {HAT } \\
\text { hprt }^{+}: \text {HAT } \\
\text { hprt }^{-}: \text {G418 } 4 \text {-TG } \\
\end{array}$ & $\begin{array}{c}10^{-3} \text { des traités } \\
1 / 75 \text { a } 1 / 500 \text { des intégrations } \\
1,4.10^{-6} \text { des traitées } \\
2,2.10^{-7} \text { des traitées } \\
10^{-3} \text { des } \mathrm{G}_{418^{r}}: 10^{-6} \text { des traitées }\end{array}$ \\
\hline $\begin{array}{l}\text { Double sélection } \\
\text { Mansour et al.[12] }\end{array}$ & $\begin{array}{l}\text { ES } \\
\text { ES }\end{array}$ & $\begin{array}{c}\text { hprt } \\
\text { int-2 }\end{array}$ & $\begin{array}{l}\text { Oui } \\
\text { Faible }\end{array}$ & $\begin{array}{l}\text { néo }+/ \text { Hsv-tk- }^{-}: \text {G418 + GANC } \\
\text { néo }+/ \text { Hsv-tk- }^{-}: \text {G418 + GANC }\end{array}$ & $\begin{array}{l}\text { 19/24 des sél. ; } 4.10^{-6} \text { des traitées } \\
4 / 81 \text { des sél. ; } 5.10^{-6} \text { des traitées }\end{array}$ \\
\hline $\begin{array}{l}\text { Amplification génique } \\
\text { Zimmer et al. [14] } \\
\text { Joyner et al. [15] }\end{array}$ & $\begin{array}{l}\text { ES } \\
\text { ES }\end{array}$ & $\begin{array}{c}\text { Hox } 1.1 \\
\text { En-2 }\end{array}$ & Faible & $\begin{array}{c}\text { PCR } \\
\text { néo+: G418 + PCR }\end{array}$ & $\begin{array}{l}1 / 150 \text { des traitées } \\
1 / 300 \text { des } G 418\end{array}$ \\
\hline $\begin{array}{l}\text { Gène de résistance } \\
\text { sans promoteur } \\
\text { Doetschman et al. [16] } \\
\text { Dorin et al.[17] } \\
\text { Sedivy et al.[18] }\end{array}$ & $\begin{array}{l}\text { ES } \\
\text { CI21 } \\
3 \mathrm{~T} 3\end{array}$ & $\begin{array}{l}\text { hprt } \\
T \text { (SV40) } \\
\text { pmt }\end{array}$ & $\begin{array}{l}\text { Oui } \\
\text { Oui } \\
\text { Oui }\end{array}$ & $\begin{array}{c}\text { néo+/hprt -: G418 + 6-TG } \\
\text { néo+ } \text { né G418 + PCR }^{+} \text {néo }: \text { G418 }\end{array}$ & $\begin{array}{c}\quad 4 / 4 \text { des sél. } ; \simeq 10^{-6} \text { des traitées } \\
\simeq 6.10^{-6} \text { des traitées }: 3 / 11 \text { des } \mathrm{G} 418^{r} \\
10^{-2} \text { des sél. }\end{array}$ \\
\hline
\end{tabular}

* Les taux sont rapportés au nombre de cellules ayant survécu au protocole de sélection (sél.) ou au nombre de cellules transfectées (traitées).

général, une intégration aléatoire du vecteur dans le génome hôte ne fournira pas de séquences activatrices et ne permettra pas l'expression de néo. A l'inverse, un événement de recombinaison homologue du vecteur avec le gène cible place le gène néo défectif sous le contrôle des séquences régulatrices du gène endogène, qui permettent son expression. La sélection s'effectue par la résistance au G418. Dans certains cas cependant, le gène néo défectif s'insère à proximité de séquences activatrices non homologues qui entraînent son expression. Cette technique de sélection ne constitue donc qu'une étape d'enrichissement qui doit être complétée par un criblage plus spécifique. Elle n'est applicable qu'à des gènes exprimés dans les cellules ES et conduit à l'introduction d'un gène étranger dans le génome receveur.

Doetschman et al. [16] ont utilisé cette stratégie avec succès pour inactiver le gène $\mathrm{Hprt}$ en insérant le gène néo au milieu de l'exon 3. Ils ont sélectionné par le G418, puis par la résultaient d'un événement de recombinaison homologue.

Dorin et al. [17] ont associé cette technique pour réaliser un enrichissement et la PCR pour détecter les recombinants homologues.

Afin d'estimer l'efficacité de cette technique d'enrichissement, Sedivy et al. [18] ont transfecté différents types de cellules avec des vecteurs contenant le gène néo sous le contrôle d'un promoteur autonome (permettant l'expression de néo quel que soit le site d'intégration) ou privé de promoteur (l'expression de néo n'est obtenue que si le vecteur s'est intégré en phase dans le gène endogène). Avec ce deuxième type de vecteur, les auteurs obtiennent environ cent fois moins de cellules résistantes au G418 que lorsque le gène possède un promoteur autonome. L'utilisation d'un gène de sélection sans promoteur multiplie donc par 100 l'efficacité de clonage des cellules ayant subi un événement de recombinaison homologue.

Une synthèse des résultats obtenus grâce à ces différentes stratégies est présentée dans le Tableau I. Il mentionne notamment la fréquence de recombinaison homologue observée par les différents auteurs.

\section{La recombinaison \\ homologue par micro-injection dans l'œuf}

Très récemment, Brinster et al. [19] ont réussi à obtenir un événement de recombinaison homologue après avoir micro-injecté dans des œufs fécondés une construction destinée à corriger la délétion d'une partie 5' du gène d'histocompatibilité $E \alpha$, présente chez certaines lignées de souris. Ils ont micro-injecté 10602 œufs et obtenu 1841 souriceaux, dont 506 avaient intégré la construction, mais un seul d'entre eux avait subi une correction de la délétion (mise en évidence par Southern blot).

Une analyse fine du gène ainsi modifié a pu montrer qu'une quinzaine de mutations ponctuelles étaient apparues au cours de cet événement de recombinaison. Si cette donnée était confirmée par d'autres travaux, elle 
constituerait un handicap sérieux pour tous ceux qui envisagent d'utiliser la recombinaison homologue pour modifier précisément la structure d'un gène.

Un autre intérêt de ce travail est d'avoir réussi à modifier un gène complètement inactif (du fait de la délétion). Cependant, et probablement à cause des néomutations observées, l'ARN, transcrit dans les tissus attendus, n'est pas traduit en protéine.

\section{Les facteurs qui influencent la fréquence de recombinaison homologue}

Lorsque l'on analyse les résultats obtenus par les différents auteurs, on constate des variations considérables du taux de cellules ayant subi un événement de recombinaison homologue par rapport aux cellules manipulées (de 6,7.10-2 [14] à 2.10-7 [11]). Les conditions expérimentales sont très différentes d'une expérience à l'autre et de nombreux paramètres sont susceptibles d'expliquer ces fluctuations. Il est donc souvent difficile de déterminer l'influence individuelle de chacun de ces facteurs. L'importance de certains d'entre eux a cependant été discutée par plusieurs auteurs, à la lumière des résultats expérimentaux obtenus dans un même laboratoire en faisant varier un seul paramètre, tous les autres étant fixés. Seules des observations de ce type permettent de tirer des conclusions de valeur, qui varient parfois selon les auteurs.

Le mode d'introduction de l'ADN exogène. Selon Thomas et al. [6], la transfection par précipitation au phosphate de calcium provoquerait une altération de l'ADN transfectant, défavorisant la recombinaison homologue (ADN introduit sous forme de concatémères, subissant fréquemment des mutations et s'intégrant souvent en des sites aléatoires). La micro-injection et l'électroporation sont les deux principales techniques employées. Il est délicat de les comparer en termes de rendement pour la recombinaison homologue. La première offre l'avantage d'un taux d'intégration très élevé (qui peut atteindre 10 à $20 \%$ ). L'électroporation présente une efficacité de $\mathrm{m} / \mathrm{s} n^{\circ} 1$ vol. 6, janvier 90 transfection moindre mais elle permet de traiter $10^{7}$ cellules en une seule manipulation. Par ailleurs, sous certaines conditions expérimentales, il est possible d'introduire dans la majorité des cellules une seule copie du vecteur.

La structure de l'ADN transfectant. Les travaux effectués chez la levure ont montré qu'un vecteur est un substrat plus efficace pour la recombinaison homologue lorsqu'il est sous forme linéaire. Les résultats obtenus par Thomas et al. [6] indiquent qu'il en serait de même dans les cellules de mammifère.

Le taux de ces recombinants homologues ne varie pas lorsque l'on fait passer de 5 à 100 le nombre de copies du vecteur introduites dans chaque cellule [6]. Aussi peut-on supposer que l'étape de reconnaissance des séquences homologues ne serait pas limitante.

La longueur des séquences homologues semble jouer un rôle important puisque, en la faisant passer de 4 à $9 \mathrm{~kb}$, Thomas et al. [10] ont multiplié par 20 le taux de recombinants homologues obtenus. Ils ont donc suggéré d'utiliser des vecteurs comportant des séquences homologues de plusieurs kilobases. Cela ne semble cependant pas indispensable puisque plusieurs auteurs ont obtenu des résultats intéressants avec des vecteurs présentant moins de $3 \mathrm{~kb}$ d'homologie avec le gène endogène $[14,15]$.

La présence de séquences homologues aux extrémités de l'ADN exogène linéarisé a un effet très controversé. Selon Smithies et al. [5], elle favoriserait clairement la recombinaison avec le gène cible. A l'inverse, Mansour et al. [12] affirment que la présence d'une large région non homologue (le gène $H s v$-tk) à une extrémité (et même aux deux) de leur vecteur n'a pas réduit la fréquence des recombinants homologues. Selon ces auteurs, la linéarisation du vecteur n'accroît pas l'efficacité de la recombinaison homologue en créant des extrémités homologues capables d'envahir la molécule cible, mais plutôt en conférant au vecteur une conformation topologiquement plus favorable.

Le rôle joué par les séquences non homologues présentes dans le vecteur a été discuté. Thomas et al. [10] recommandent de réduire au maximum la longueur de ces séquences. Celle-ci est toutefois supérieure à $1 \mathrm{~kb}$ avec les stratégies utilisant un gène de résistance $[10,12,15,16,18]$. La fréquence de recombinaison homologue très élevée observée par Zimmer et al. [14] pourrait être en partie due à l'utilisation d'un vecteur dans lequel la région non homologue a été réduite à 20 paires de bases. Thomas et al. [6] ont étudié l'influence de la nature de la modification à réaliser (insertion, délétion, mutation ponctuelle) et ont conclu que la recombinaison homologue semble privilégier l'introduction de petites insertions.

Enfin, certains auteurs préconisent de purifier l'ADN transfectant pour éliminer les séquences plasmidiques [10, 18], alors que, selon d'autres, la présence de ces séquences n'est pas gênante [11].

Facteurs liés au locus cible. L'influence de la localisation du locus cible dans le génome a été discutée à la suite d'expériences dans lesquelles des lignées cellulaires possédant le gène cible à des localisations différentes ont été transfectées avec le même vecteur. Thomas et al. [6] ont constaté que la localisation du gène cible n'influençait pas sensiblement le taux de recombinaison homologue, contrairement à Song et al. [8], qui lui attribuent une part des différences observées.

Le niveau d'expression du gène à modifier pourrait également intervenir, puisque la conformation de la chromatine n'est pas la même lorsqu'un gène est exprimé et lorsqu'il est silencieux. Mansour et al.[12] avancent cette hypothèse, parmi d'autres, pour expliquer l'obtention d'un plus grand nombre de recombinants homologues avec le gène $\mathrm{Hprt}$ (exprimé dans les cellules ES) qu'avec le gène int-2 (très faiblement exprimé). L'influence de ce facteur est certainement limitée. En effet, Smithies et al. [5] ont obtenu des recombinants homologues au locus $\beta$-globine, que ce gène soit exprimé ou non dans les cellules receveuses* (voir note page 28).

En conclusion, les remarques émises par les différents auteurs sont souvent contradictoires et il est bien difficile d'énumérer les facteurs qui font réellement varier la fréquence de la 


\section{Mécanismes moléculaires de}

La recombinaison homologue est un phénomène d'échange de matériel entre deux molécules d'ADN de séquence très voisine au cours duquel aucun nucléotide n'est perdu ni ajouté.

$D$ ans un premier temps, les molécules mises en jeu s'apparient ba'se à base dans la région d'homologie. La molécule intermédiaire, qui réunit deux double brins d'ADN recombinants, contient quatre brins d'ADN échangeant leurs appariements au point de contact. Cette structure en brins croisés est appelée " structure de Holliday " (figure IA). L'existence de cette espèce moléculaire a été suggérée par la mise en évidence de régions de double brins hétérologues, formées de deux monobrins qui ne sont pas exactement complémentaires, dans les deux molécules d'ADN issues d'une recombinaison. Un modèle moléculaire a permis de mettre en évidence deux propriétés physiques de la structure de Holliday: la migration des branches et l'isomérisation.

La rotation du squelette de la molécule permet aux monobrins croisés de passer librement d'une hélice d'ADN à l'autre. Le point d'intersection entre les deux hélices peut donc se déplacer aisément grâce à un mécanisme de fermeture à glissière au cours duquel des bases équivalentes des deux molécules originelles sont échangées: c'est le phénomène de "migration des branches " ou de "diffusion rotatoire " (figure IB). Une région d'une molécule d'ADN donnée peut donc se déplacer d'une hélice à l'autre en produisant des double brins hétérologues plus ou moins étendus. Des brins peuvent ainsi être échangés entre deux double brins homologues d'ADN à une certaine distance de la cassure originelle.

La seconde propriété physique de la structure de Holliday est son aptitude à subir une isomérisation (figure IIA). Un simple réarrangement stérique (deux rotations successives de $180^{\circ}$ d'une moitié du complexe moléculaire autour du point de croisement) place les brins croisés "à l'extérieur » de la molécule et croise les brins non recombinés (figure IIB).

L'ultime étape de la recombinaison homologue est la résolution de la structure de Holliday en deux molécules double brins. Ce phénomène met en jeu des nucléases et engendre deux types de paires de double brins: des double brins non recombinants qui n'ont échangé qu'une région monobrin (sans isomérisation de la structure de Holliday) et des double brins recombinants qui résultent d'un échange des quatre brins (après isomérisation de la structure de Holliday).

Des études menées chez la levure ont montré que la recombinaison n'est initiée que si une des deux hélices d'ADN présente une discontinuité : cassure ou région de séparation des deux brins. Deux modèles ont été proposés, toujours chez la levure, afin d'expliquer la for- mation de la structure de Holliday. Le premier modèle a été établi par Meselson et Radding en 1975 et suggère que la recombinaison est initiée par une cassure simple brin de l'un ou l'autre des double brins impliqués (figure III). L'extrémité 3' du brin interrompu est utilisée comme amorce de replication par l'ADN polymérase. Cette néosynthèse implique un déplacement de l'extrémité 5' du brin, qui envahit alors l'autre double brin dans la région d'homologie et donne naissance à une structure tricaténaire $(A)$. Le brin envahisseur du double brin donneur déplace le brin homologue du double brin receveur, et la région double brin hétérologue est étendue grâce à la synthèse du brin donneur, concomitante de la dégradation du brin homologue déplacé du double brin receveur(B). La structure de Holliday est engendrée par ligation des extrémités 3' du brin néosynthétisé et 5' du brin dégradé $(C, D, E)$.

Le second modèle a été proposé par Szostak et al. en 1983 (figure IV). Il implique la double cassure d'un des deux double brins et la création, par des exonucléases, d'une délétion bordée d'extrémités 3' simple brin. Une des extrémités 3' envahit l'autre double brin $(A)$, est utilisée comme amorce de replication pour l'ADN polymérase et déplace le brin homologue de la molécule acceptrice $(B)$. La synthèse d'ADN à partir des deux extrémités 3' de la molécule donneuse permet de combler la délétion $(C)$. Le phénomène de migration des branches engendre deux jonctions de Holliday qui sont ensuite résolues par l'action d'exonucléases (D).

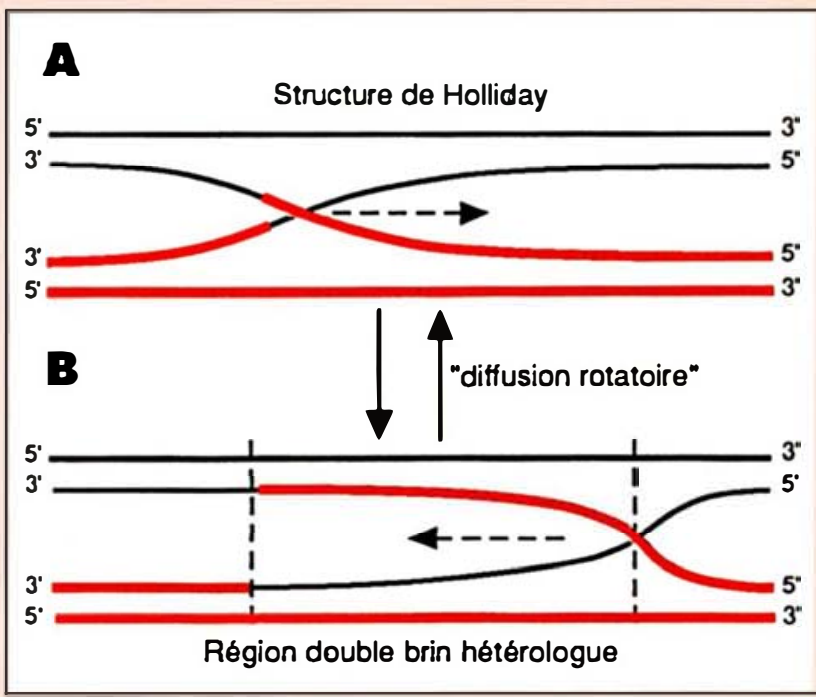

Figure I. Diffusion libre du point de jonction entre deux hélices d'ADN recombinantes. 


\section{la recombinaison homologue}

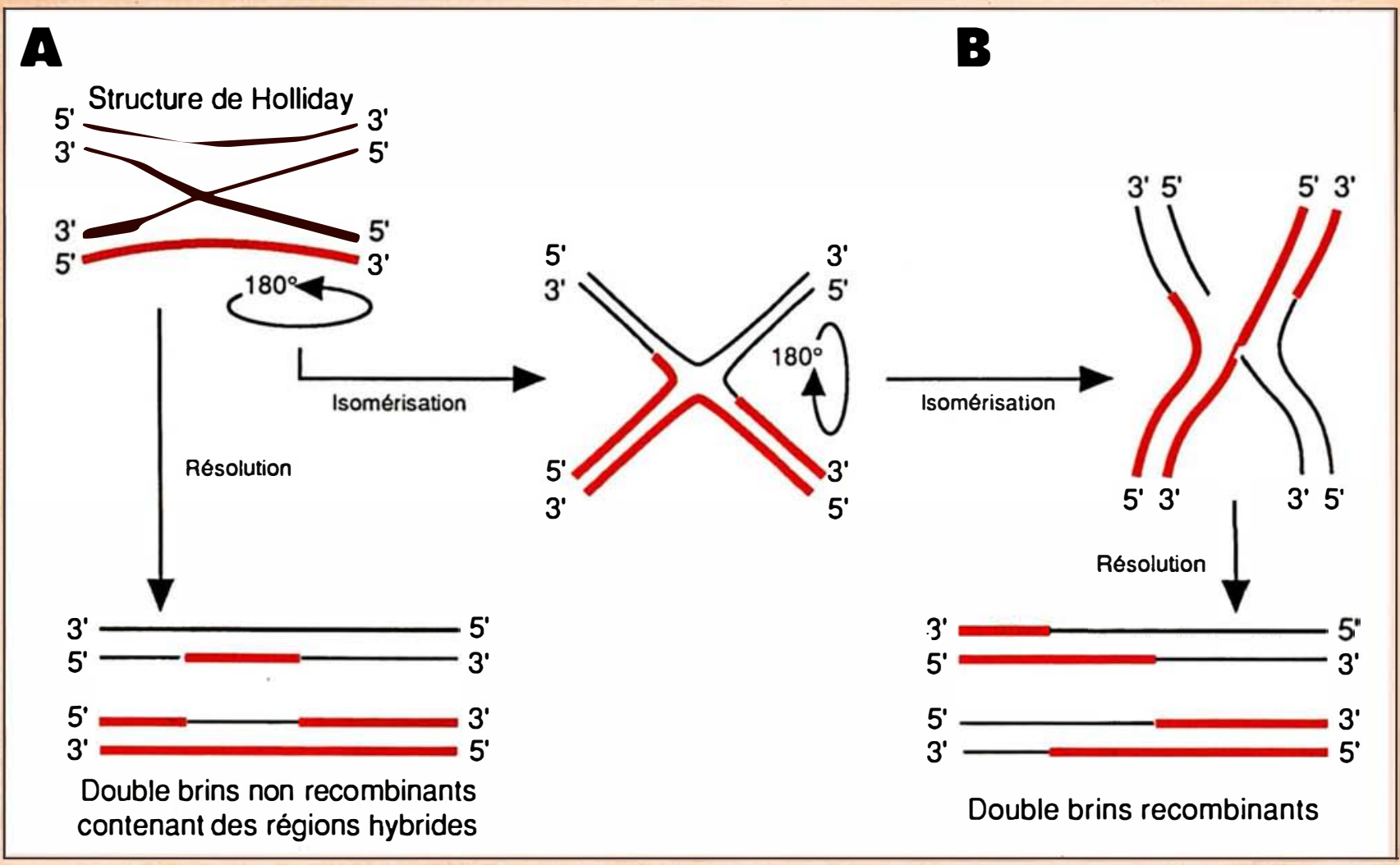

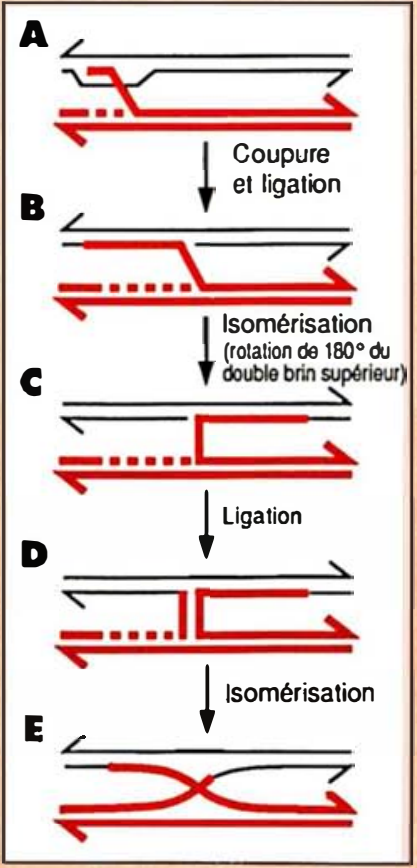

$m / s n^{\circ} 1$ vol. 6, janvier 90
Figure II (en haut). Isomérisation et résolution de la structure de Holliday.

Figure III (à gauche). Le modèle de Meselson-Radding : formation de la structure de Holliday par cassure simple brin.

Figure IV (à droite). Le modèle de Szostak et Orr-Weaver : formation de la structure de Holliday par cassure double brin.

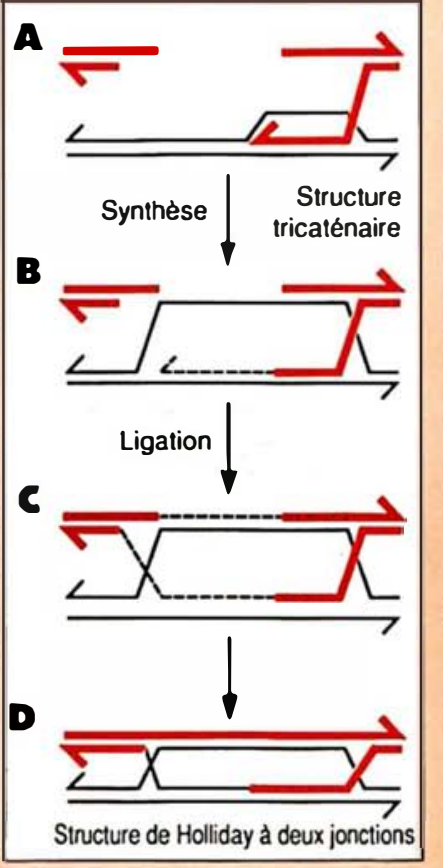

Structure de Holliday à deux jonctions 
20. Baker MD, Shulman MJ. Homologous recombination between transferred and chromosomal immunoglobulin kappa genes. $\mathrm{Mol}$ Cell Biol 1988; 8 : 4041-7.
* Note de la rédaction: la possible recombinaison homologue avec des gènes non exprimés a été confirmée récemment avec deux nouveaux exemples (Science 1989 ; 245 : 1234 6). recombinaison homologue. Seules peuvent être comparées les observations effectuées dans les mêmes conditions expérimentales et le rapprochement des résultats obtenus par différents laboratoires doit être considéré avec beaucoup de prudence.

\section{La précision du mécanisme}

Le résultat attendu lors d'un événement de recombinaison homologue est le remplacement des séquences endogènes par les séquences exogènes homologues (voir encadré, pages 26, 27), sur les mécanismes moléculaires de la recombinaison. Les résultats publiés dans la littérature incitent à penser qu'il s'agit d'un mécanisme relativement fréquent, mais qui n'est pas forcément exclusif. Plusieurs auteurs ont en effet obtenu des cellules portant, en plus de la modification attendue, des mutations ponctuelles [19], des insertions [7], ou des réarrangements complexes [1 1, 18]. La fréquence de ces modifications est d'ailleurs sous-estimée, car la plupart des auteurs ont réalisé une analyse par restriction des clones recombinants qui ne permet pas de mettre en évidence des petites insertions, délétions, ou des mutations ponctuelles, si celles-ci ne font pas apparaître ou disparaître un des sites de restriction analysés. Seule une séquence complète de la région ciblée peut rendre compte de toutes les modifications introduites. Il serait intéressant que des études comparables à celles réalisées par Brinster et al. [19], démontrant le nombre important de mutations ponctuelles dans un transgène intégré par recombinaison homologue, soient menées pour savoir si les imprécisions de ce mécanisme constituent un phénomène accidentel ou, au contraire, assez fréquent.

\section{Applications et perspectives}

être connue

Une transgénèse dirigée. Jusqu'ici, les différentes techniques utilisées en transgénèse conduisaient à l'intégration, à un ou plusieurs sites non prévisibles, d'un nombre variable de copies du fragment d'ADN introduit. La recombinaison homologue permet de modifier très précisément une région chromosomique donnée. Le ciblage obtenu est parfait (à une base près) et la mutation introduite peut porter sur une seule base. Cependant, les résultats obtenus par certains auteurs indiqueraient, s'ils étaient confirmés, que le mécanisme n'est pas toujours aussi précis, puisque de courts remaniements, ainsi que des mutations ponctuelles, peuvent apparaître au cours de l'événement de recombinaison.

La thérapie génique somatique. Le remplacement d'un gène défectueux par son homologue fonctionnel conduit à entrevoir la possibilité de guérir définitivement certaines maladies génétiques. La moelle osseuse constitue le tissu privilégié pour ce type de manipulations en raison de la possibilité de réinjecter à un individu ses propres cellules souches hématopoïétiques modifiées génétiquement in vitro, supprimant par là tout risque de rejet immunologique. Les cellules somatiques seraient ainsi corrigées, sans atteinte des cellules germinales*.

La création de modèles animaux de maladies humaines génétiquement déterminées. Plusieurs stratégies pourront être utilisées pour modifier la structure d'un gène chez la souris et en comprendre la régulation. Il sera possible d'inactiver un gène en altérant sa séquence codante ou ses éléments de régulation, ou de réaliser une mutagénèse très précise. Le remplacement d'un gène normal de souris par le gène homologue humain défectueux permettra de reproduire chez la souris des modèles d'une maladie génétique humaine (avec la même altération génétique). Des équipes tentent actuellement d'obtenir par recombinaison homologue des modèles animaux des déficits en adénosine déaminase (ADA) ou en donné dans un sens prédéterminé ouvre de très larges horizons dans des domaines variés. Nous nous contenterons de citer ici cinq applications potentielles. Rappelons que le gène cible doit avoir été cloné et qu'au moins une partie de sa séquence doit
* Note de la rédaction: cette perspective est actuellement obscurcie par l'insuffisance des connaissances sur la cellule souche médullaire, qui, en culture, perd rapidement ses propriétés. 
nucléoside phosphorylase (NP), tous les deux responsables chez l'homme d'une absence de défenses immunitaires.

L'étude de gènes impliqués dans le développement. La même stratégie sera mise en ouvre pour étudier le rôle de gènes potentiellement impliqués dans le développement. Les gènes homéotiques seront certainement des candidats privilégiés pour ce type d'expériences [13, 14].

La production d'anticorps monoclonaux humains. Un événement de recombinaison homologue peut être utilisé pour modifier la spécificité des gènes d'immunoglobulines d'un hybridome, afin de produire des molécules d'immunoglobulines «hybrides », ainsi que l'ont montré récemment Baker et Schulman [20]. Il est donc envisageable de pouvoir produire des anticorps monoclonaux «humains», en remplaçant la région constante des gènes d'immunoglobulines d'un hybridome murin par la région homologue humaine. L'utilisation de telles molécules supprimerait d'emblée les réactions isotypiques liées à l'introduction d'anticorps monoclonaux murins chez l'homme.

\section{Conclusion}

La recombinaison homologue est certainement le procédé de mutagénèse et de transgénèse le plus précis que l'on puisse imaginer à l'heure actuelle. Elle permet de réaliser des modifications extrêmement précises d'un gène. Toutes les étapes du protocole de transgénèse par modification des cellules ES ne sont pourtant pas encore parfaitement maîtrisées. Si plusieurs auteurs ont réussi à obtenir des cellules ES recombinées et des animaux chimériques, peu d'entre eux ont pu produire des lignées transgéniques stables, notamment en raison d'un très faible taux de chimérisme dans la lignée germinale.

Malgré les difficultés techniques qu'il reste à résoudre, on peut aujourd'hui prédire que les domaines d'application de la recombinaison homologue ne cesseront de s'étendre, notamment dans l'espoir de guérir un jour, par thérapie génique somatique, certaines maladies humaines graves telles que les thalassémies ou la drépanocytose

$m / s n^{\circ} 1$ vol. 6, janvier 90

\section{Summary}

Homologous recombination : new approaches to transgenesis in mammals

Homologous recombination is a new technique for transgenesis in mammals using embryonic stem cells in which a foreign DNA fragment replaces the homologous sequence in a recipient genome, introducing or correcting a specific mutation in a given gene. Modified embryonic stem cells can be injected into mouse embryos to produce transgenic animals. This technique is, by far, the most powerful one able to achieve gene targeting. We present several strategies which have been recently developed in order to select cells which have undergone the expected homologous recombination event with foreign DNA. We also discuss several factors which could affect targeting efficiency. This new technology will be extensively used in the future, owing to the numerous applications of gene targeting in the biomedical sciences : transgenesis in mice or other mammals, gene therapy to repair deleterious mutations, creation of animal models for human genetic diseases, study of genes that control development and the production of human monoclonal antibodies.

\section{TIRÉS A PART}

X. Montagutelli. 